\title{
Gestión de la Comunicación Intercultural desde el Diálogo de Saberes y Haceres
}

\author{
Management of Intercultural Communication from \\ the Dialogue of Knowledge and Doings
}

Yulmar Runel Montoya Ortega ${ }^{1}$

\section{Resumen}

La Gestión de la Comunicación Intercultural juega un papel fundamental y estratégico en el Diálogo de Saberes y Haceres, porque armoniza, comparte y construye colectivamente. Por ello, es importante compartir y describir los procesos que se vivencian desde la comunicación occidental y la comunicación intercultural y la apuesta en la gestión de una comunicación más humana. La comunicación intercultural es un elemento en construcción, que ha venido ganando terreno por su forma de abordar y humanizar las buenas prácticas que abonan a un mundo mejor e incluyente. La comunicación intercultural tiene que ver con aspectos de propiciar relaciones interpersonales y colectivas, donde comparten conocimientos y saberes a través de la práctica, la hermandad y la reciprocidad. En los diálogos de saberes y haceres quienes proponen y hacen son los mismos pueblos, son las comunidades indígenas y afrodescendientes, a través de sus vivencias y realidades, a través de sus procesos de construcción propia, de sus familias y comunidades, con el fin de aportar y crear espacios de visibilización y construcción participativa.

Palabras clave: Comunicación, Intercultural, Diálogo, Saberes, Haceres

\section{Abstract}

The Management of Intercultural Communication plays a fundamental and strategic role in the Dialogue of Knowledge and Facts Doings, because it harmonizes, shares and builds collectively. Therefore, it is important to share and describe the processes that are experienced from Western communication and intercultural communication and the commitment to the management of a more human communication. Intercultural communication is an element under construction, which has been gaining ground due to its approach and humanization of good practices that contribute to a better and inclusive world. Intercultural communication has to do with aspects of the promotion of interpersonal and collective relationships, where they share knowledge and knowledge through practice, brotherhood and reciprocity. In the dialogues of knowledge and doers that they propose and do are the same peoples, they are the

1 Máster en Comunicación Intercultural con enfoque de género. Director del Instituto para la Comunicación Intercultural de la Universidad de las Regiones Autónomas de la Costa Caribe Nicaragüense. Correo: yulmar.montoya@uraccan.edu.ni (D): https://orcid.org/oooo-0003-1873-2026

Recibido: 03/03/2019 Aprobado: 05/05/2019

Montoya-Ortega, Y. (2019). Gestión de la comunicación intercultural desde el diálogo de saberes y haceres. Revista Electrónica de Conocimientos, Saberes y Prácticas, 2(1), 8-20. D0l: https://doi.org/10.5377/recsp.v2i1.8163 
indigenous and afro-descendant communities, through their experiences and realities, through their own construction processes, their families and communities, in order to contribute and create opportunities for visibility and participatory construction.

Key Word: Communication, Intercultural, Dialogue, Knowledge, Doings

\section{Introducción}

Por años se han construido diferentes perspectivas y opiniones respecto a la comunicación como teoría. Pero lamentablemente gran parte de la sociedad no ha sido lo suficientemente capaz de interiorizar en las corrientes que se pueden interpretar y desprender de ella. Históricamente el ser humano se ha centrado en comprender la comunicación únicamente como la forma de dialogar entre un individuo y otro, es decir, un emisor-mensaje-receptor, que emite una codificación unidireccional. Pero, no la han dimensionado como un aspecto a profundidad con espíritu vivencial, de derechos y reciprocidad, es decir, una comunicación más humana y de gestión.

Con los años, la comunicación como tal se ha percibido de diferentes formas, pero también se ha concebido como el propio sistema de mensajes o informaciones, entre personas físicas o sociales, a través de medios personalizados o de masas, mediante un código de signos también convenido o fijado de formas arbitraria, (Rizo, 2004).

En la actualidad existen muchas vertientes en torno al tema en cuestión, por ejemplo, la comunicación occidental y la comunicación Intercultural. Ambas tienen sus semejanzas respecto al compartir e interactuar, pero son totalmente diferentes, puesto que una se dedica más a los criterios establecidos por las grandes corrientes divergentes de un mundo altamente globalizado, sollamado y desvariante por su verticalidad. Ante ello, es esencial conocer un poco las vicisitudes y las vivencias que les caracterizan tomando en consideración el Diálogo de Saberes y Haceres, como un elemento fundamental para el compartir la palabra, los conocimientos, saberes y prácticas desde lo comunitario y lo occidental. El presente artículo describe esos procesos que tejen una comunicación intercultural humana, de derechos humanos que se vivencian a través de diálogos interculturales y esa relación en armoniosa entre los distintos cultivos y siembras que se materializan por medio del diálogo de saberes y haceres.

\section{Metodología}

La identificación de autores que resaltan y abordan esta temática, fue clave para la construcción del presente artículo, porque permitió socializar, debatir y reforzar los distintos planteamientos compartidos durante todo lo descrito. Este ejercicio permitió la revisión documental para fortalecer más el aporte del escrito a través de consultas de documentos escritos por Alta Hooker, Yulmar Montoya, José Atupaña, Asunción 
Lande, Miquel Alsina, Martha Rizo, Erik Torrico, Harold Lasswell, Aurelio Ríos y Bolivar Yantalema, especialistas en comunicación intercultural y educación, quienes además de escribir en este ámbito aportan a la conceptualización de la interculturalidad desde distintas miradas. En la búsqueda de la literatura se incluyó temática referidas a la comunicación occidental, comunicación intercultural, interculturalidad y diálogo de saberes y haceres.

\section{Criterios de inclusión y exclusión}

En la revisión bibliográfica se incluyó escritos elaborados por cada uno de los autores antes mencionados, que permitió fortalecer los planteamientos como un aporte a la construcción del presente artículo desde donse se hace un análisis crítico y propositivo que destaca las características particulares de la comunicación occidental y la comunicación intercultural. Sobre las revisiones sistemáticas y los estudios científicos se aplicó como criterio de inclusión que los escritos abordarn aspectos sustanciales sobre la comunicación occcidental, la comunicación intercultural, interculturalidad y diálogo de saberes y haceres. El único criterio de exclusión fue que los artículos no incluyeran información sobre la temática referida que se aborda en el presente escrito.

\section{Fuentes de datos}

De un total de 20 escritos, referencié artículos que aportan a la temática desarrollada en este artículo. Para proceder a la selección se revisaron los artículos completos, con el fin de analizar, criticar y decidir por la información necesaria y que estaba relacionada con el objetivo de este escrito.

\section{Análisis de los Datos}

La información analizada se estructuró en tres espacios: La Comunicación Occidental y la Comunicación Intercultural; Gestión y Comunicación Intercultural; La comunicación y el diálogo de saberes y haceres; El cuido y uso adecuado de la información; Una comunicación solidaria; Características de la comunicación; Hace visible lo históricamente invisible. Finalmente, toda la información extraída, se revisó sobre autoría, año, finalidad y fuentes de información.

De los artículos originales se obtuvo información sobre autoría, libros, tesis y revista en la que han sido publicados y año de publicación, país donde se realizó el estudio, tipo de estudio, sujetos, origen y conclusiones. 


\section{Resultados y Discusión}

\section{La Comunicación 0ccidental y la Comunicación Intercultural}

Es fundamental afirmar que tanto la comunicación occidental como la comunicación intercultural, tienen sus propias características y sus propias bondades. La occidental es utilizada más por las grandes masas corporativas, que se traslapan a través de los medios de comunicación masivos y las políticas hegemónicas orientadas por diversos sectores, entre estos, políticos y empresarios, que la convierten y amoldan según sus intereses, generando así una comunicación lineal y operativa que responde a la clase oligarca, y este tipo de comunicación facilita información entre elites y sus poderíos, Lasswell (1985), mas no a los intereses de los sectores vulnerables e históricamente invisibilizados.

Las iniciativas y procesos implementados por la comunicación occidental nacieron con el interés de realizar exámenes a las iniciativas relacionadas con la interrelación y su expresividad, pero sin el interés de entenderla como una forma de vida. Esta emergió en Occidente en el siglo XX con la impronta del saber empírico, mensurable y aplicable, y vinculado a los intereses políticos y económicos del capitalismo y se concibe, ante todo, como recurso instrumental, de apoyo a intereses de poder, (Torrico, 2015, p. 4 y 5).

Este tipo de ejercicios limita a la sociedad a poder desarrollar y convivir una comunicación más acorde a las realidades cotidianas, puesto que prevalece la impunidad y la corrupción sobre todas las cosas. Hoy por hoy, no existe un compromiso que de salida a que la hegemonía se dé la oportunidad de desaprender para retomar actitudes colectivas por el bien común. Pues la comunicación como espacio de compartir es un elemento necesario para encontrar respuestas a los diversos problemas que enfrenta la humanidad, por los grandes intereses de pocos, donde prevalece el amarillismo y la contaminación publicitaria, creando incluso la nota roja para vender y satirizar las diferentes formas de vida, de quienes luchan día a día por enfrentar las realidades desiguales.

Con el tiempo, se ha interpretado la necesidad de implementar una comunicación más humana y pertinente, que aporte al mundo de la colectividad desde la diversidad, unidad y hermandad. Una comunicación diferente que abone a generar espacios de sinergia que apunten al buen caminar y a la organización colectiva desde diversas instancias y organizaciones, es posible. En este sentido uno de los grandes avances de la comunicación es la fuente de nuevas comunicadoras y comunicadores con un pensamiento crítico, más humano y de consenso que considera las bases y los acontecimientos para darle un tratamiento positivo a la información que abona a las buenas prácticas de diversos sectores y organizaciones. 
No se puede obviar que el mundo moderno con sigo mismo ha traído un sin número de herramientas que posibilitan realizar un trabajo con mayor escala y en este sentido las Nuevas Tecnologías de la Información y Comunicación (Tics), permiten llegar a un sin número de espacios físicos y digitales. Pero también la evolución en la formación de profesionales en la materia, puesto que con el pasar de los años, la academia ha venido creando nuevas perspectivas y adaptaciones, ejemplo; de periodismo pasamos a comunicación social y de esta a comunicación para el desarrollo, pero en realidad todos estos nuevos nacimientos no tienen un horizonte claro, es más de lo mismo, porque las brechas y desigualdades siguen creciendo.

A pesar de todo ese esfuerzo, aún es posible aportar a un ejercicio diferente del abordaje e implementación de la comunicación, creando espacios formativos y de conciencia, para realizar una gestión pertinente de la misma para aportar a la construcción de ciudadanías interculturales. Muy a pesar de ello, es necesario considerar que no todo está perdido, pero que si es importante que los nuevos talentos humanos tomen en cuenta que es vital aprender nuevas herramientas que aporten a la armonía, el Buen Vivir y el Bien Hacer. Ha habido un sector históricamente invisibilizado y estos son los pueblos indígenas y afrodescendientes.

Uno de los grandes hitos históricos es el nacimiento de la Comunicación Intercultural, como una propuesta vital para visibilizar lo históricamente invisibilizado, desde un currículo pertinente creado para aportar y fortalecer los procesos implementados desde los pueblos indígenas y afrodescendientes, que aporte a la construcción de ciudadanías interculturales desde el abordaje de una comunicación más humana y complementaria. La Universidad de las Regiones Autónomas de la Costa Caribe Nicaragüense (URACCAN), ha creado este tipo de propuestas y que hoy por hoy ha graduado una cantidad considerable de profesionales en la materia para aportar a ese buen hacer para aportar al Buen Vivir y el Vivir Bien. Los programas de formación partieron de técnico medio, licenciaturas y finalmente al grado de Maestría.

\section{Gestión y Comunicación Intercultural}

Si bien es cierto, la comunicación intercultural es un elemento en construcción, que ha venido ganando terreno por su forma de abordar y humanizar las buenas prácticas que abonan a un mundo mejor e incluyente, que aporta a la organización y a construir en colectivo, fortaleciendo así los planes de vida de los pueblos, pero además permite practicar una comunicación horizontal, colectiva y de respeto entre la diversidad de pensamiento, en armonía con la Madre Tierra y el Buen Vivir.

En este sentido la formación comunicadora y comunicadores interculturales, es una oportunidad sine qua non para la sociedad, considerando que son actores que aportan a una gestión pertinente de la comunicación desde el abordaje de esta, y la visibilización, partiendo de las realidades, derechos colectivos e individuales de los 
pueblos, que no transgrede, sino que abona a la solución de problemas cotidianos de manera incluyente y respetuosa. Las comunicadoras y comunicadores formados en este contexto aprenden a trabajar de manera respetuosa y consensuada, aportando a la solución de problemas a través de el abordaje de una comunicación pertinente, respetuosa y de igualdad, que no denigra y ni es racista.

La comunicación intercultural, es un elemento que respeta, cuida y aporta a la solución de problemas cotidianos, que además busca una solución a las problemáticas que enfrentan los pueblos y la sociedad en general. Las buenas prácticas de la comunicación intercultural se materializan a través de diversos aspectos, entre estos, la oralidad, los saberes y haceres y el compartir la información de manera responsable con miras a la armonía y el vivir con dignidad. Además, esta se fundamenta desde el derecho humano de los Pueblos Indígenas y Afrodescendientes desde la visión cosmogónica que practican partiendo de sus realidades.

\section{La comunicación intercultural y el diálogo de saberes y haceres}

La comunicación intercultural tiene que ver con aspectos de propiciar relaciones interpersonales y colectivas, donde comparten conocimientos y saberes a través de la práctica, la hermandad y la reciprocidad. Los pueblos indígenas comparten sus saberes a través de la oralidad y los haceres a través de la práctica, por ejemplo, las siembras y cosechas. Asimismo, significa diálogo en igualdad de condiciones a fin de establecer relaciones interculturales.

"Cada pueblo tiene su propia manera de entender el mundo, su propia cosmovisión, su forma de sentir, de querer, de bailar, de expresarse, haciendo de la Comunicación Intercultural un elemento de unidad en la diversidad. El papel fundamental de la Comunicación Intercultural parte de la espiritualidad y de construir puentes de confianza. Debe aportar a hacer visible la filosofía y la cosmovisión de vida de los pueblos, la salud física, mental y espiritual de la mujer, el hombre, la familia y la comunidad en armonía con el cosmos, la naturaleza y los espíritus ancestrales", (Hooker, 2017).

Por ello, la comunicación intercultural construye y crea armonía entre individuos y sectores, puesto que se trata de un aporte, de un elemento fundamental, para acompañar y aportar a la creación de buenas prácticas, de fundamentos de vida.

La comunicación intercultural ve más allá de la transmisión de información, trata de penetrar en el alma, en los corazones y en los comportamientos de los actores, para que ellos mismos creen esos procesos de Buen Vivir/Vivir bien, es un lazo equitativo y en igualdad de condiciones que permite la interrelación y la construcción de ciudadanías comprometidas con ellos mismos, las comunidades y el cuido de la Madre Tierra. 
Los diálogos comunitarios de saberes van más allá de la simple verbalización de las ideas, es la articulación de la oralidad con la práctica o los haceres (Atupaña, 2018). Entonces, la comunicación intercultural es esa herramienta que va más allá del decir, es el hacer, y hacer desde y para los pueblos indígenas.

En los diálogos de saberes y haceres quienes proponen y hacen son los mismos pueblos, son las comunidades indígenas y afrodescendientes, a través de sus vivencias y realidades, a través de sus procesos de construcción propia, de sus familias y comunidades, con el fin de aportar y crear espacios de visibilización y construcción participativa.

El diálogo de saberes haceres, se concentra en ese intercambio de conocimientos y saberes, que se materializa desde la oralidad y el compartir la palabra desde un diálogo intercultural, abierto y sincero desde el corazón, que comparte el conocimiento desde los haceres comunitarios, que se transmiten desde la siembra de la semilla y el corte de las cosechas, que luego alimenta, comparte con la colectividad y guarda para la siguiente cosecha. Asimismo, este representa el compartir conocimientos que parte de las vivencias mismas de los pueblos indígenas y afrodescendientes desde diferentes planos de la vida; comunitario, espiritual, organizacional, político, ancestral, Madre Tierra y territorio. Todo esto se encamina en la búsqueda y consolidación del Buen Vivir y el Vivir Bien. Y además permite esa relación e intercambio de conocimientos desde el plano comunitario intercultural y el llamado occidental.

El diálogo de saberes y haceres, comprende una práctica (...) de compartir aprendizajes, sabidurías, conocimientos y haceres que responden a las necesidades del buen vivir personal, familiar y comunitario, que se realiza a través de la conversación oral, en la que dos o más dialogantes o actores del diálogo sustentan ideas de pensamiento y corazón de manera amistosa, libre y rotativa con o sin la presencia de un mediador, donde prevalece el valor de la palabra y la escucha respetuosa, (Yantalema, 2016).

\section{El cuido y uso adecuado de la información}

La comunicación intercultural no puede darse sin interacción, puesto que busca universalidad y simetría entre los diferentes sectores, busca el reconocimiento de las "otras" prácticas, busca un equilibrio entre las diversas culturas, prácticas y conocimientos, es por ello, que la información debe darse como un proceso inclusivo e incluyente, que aporte y no destruya, un proceso armonioso y lleno de tolerancia y solidaridad para todos los involucrados.

"Pero no hay que conocer sólo otras culturas, sino que la comunicación intercultural implica también una toma de conciencia de la propia cultura. En muchas ocasiones nuestras comunicaciones están llenas de valores que transmitimos sin ser apenas 
conscientes de ello. La comunicación intercultural no sólo supone comunicarse con otras culturas sino también hacer el esfuerzo de repensar la propia cultura" (Alsina, 1999).

Un de las principales virtudes que juega la Comunicación Intercultural, es que aporta a esa comunicación humana, que debe de cuidar, proteger la información, que aporte a una solución de diversas realidades de manera armoniosa, evitando lastimar. Además, no agrede, no es racista ni excluyente ante los pueblos. En la realidad hay muchos actores que comercializan y descontextualizan la información para vender y hacer bocadillos a costillas de sectores desprotegidos tergiversando la noticia. En la nueva era hay muchos medios de comunicación que han satirizado las formas propias de comunicación y espiritualidad de los pueblos tachándolas de brujería, mas no entienden que para la cosmovisión indígena y afrodescendientes tienen un significado fundamental porque es de aquí donde parte la vida misma; desde esa armonía con la madre naturaleza y todos los seres vivos. Este tipo de accionar rompe los esquemas $\mathrm{y}$ formas vivenciales de quienes tienen su propia forma de entender el mundo, y este tipo de actuar solo excluye, discrimina y destruye.

Alsina (1999), añade que "en la actualidad se apuesta por la interculturalidad que supone una relación respetuosa entre culturas”. Este respeto desemboca en prácticas equitativas entre individuos, familias, comunidades, ciudades, regiones, países y el mundo, para crear sociedades llenas de amor por las otras culturas, que conozcan y respeten a los otros, que vean en otras culturas, la importancia que ven en la propia.

La Comunicación Intercultural, debe ser la interlocución de los signos, mensajes y señales de la Madre Tierra para mantener una relación de equilibrio y armonía. Comprende caminar el mandato natural que nos compromete como sus hijos e hijas. Es la comunicación de la naturaleza que se refleja en el canto de los pájaros, el aullido de los perros, el movimiento de los peces y el aleteo de las tortugas que nos avisan cuando el peligro asecha a la comunidad, el diálogo entre las montañas y los lagos, el susurro de los vientos en los idiomas que sirven como puente para la trasmisión de generación a generación de conocimientos y sabidurías ancestrales, (Hooker, 2017)

La comunicación intercultural no es aquella que discrimina, sino que establece los distintos puentes donde se fortalece la comunicación entre los distintas corrientes de la sociedad y del mundo, así pues, es un elemento comprometido con la vida, las buenas prácticas que cultivan la armonía con la Madre Tierra y el Buen Vivir de los pueblos, lo que a su vez genera una universalidad de conocimientos saberes y prácticas que fortalezcan la igualdad, la equidad y el espíritu de hermandad entre los diferentes pueblos y comunidades del mundo. 


\section{INVESTIGACIONES EDUCATIVAS}

\section{Una comunicación solidaria}

La comunicación intercultural es un proceso de solidaridad, complementariedad y respeto. Cuando alguien de la comunidad está enfermo, todos sus miembros comienzan a compartir información para aportar. Asimismo, cuando la gente toca las campanas, ese sonido, trasmite un mensaje a la población y dependiendo de ello, significa un indicador que transmite cierta comunicación sobre algún acontecimiento que está por llegar o que está sucediendo.

La comunicación intercultural va más allá de la transmisión de mensajes verbales, contiene los estilos de vida de los pueblos, sus prácticas, sus vidas mismas, su entorno, sus lazos de hermandad, la manera en que responden a las adversidades, como personas y como comunidad.

La comunicación intercultural pues, es una manera de expresarse, incluye los procesos de adaptación de los pueblos indígenas, su color de piel, sus maneras propias de reconocerse, incluye su visión del mundo y de cada elemento vivo, los pueblos indígenas se comunican no solo a través del habla, sino a través del espíritu, a través de gestos, de sonidos del viento, ayudando al otro. Evidentemente cuantos más elementos las comunidades de vida compartan más sencilla será la comunicación entre ellas, (Alsina, 1999).

\section{Características de la comunicación}

La comunicación occidental tiende a generar tipos de información para distintos sectores que están vinculados entre sí. Asimismo, es mercantil y deshumana, obedece a los grandes emporios económicos que no escatiman consecuencias y buscan el bienestar y el saqueo en sociedad con los poderes políticos inventados por la sociedad. Además, discrimina, agrede y destruye toda relación con la madre tierra. Desde su implementación, desinforma y no educa. Destruye toda soberanía alimentaria, el Buen Vivir, el Vivir Bien y aporta a la proliferación de las transnacionales como su principal arma para continuar con el exterminio y deterioro de las formas tradicionales de ver y entender el mundo.

En cambio, la comunicación intercultural; es una comunicación que informa a toda la comunidad sobre los acontecimientos, pero sin hacer discriminación. Esta propicia espacios de relaciones dialógicas entre todos los actores y no trabaja en crear discrepancias sociales, étnico culturales, no genera y no propicia relaciones de discriminación y racismo, sino que aboga por la construcción de ciudadanías interculturales, donde cada uno tiene su espacio para exponer su punto de vista y el otro recibe sin discriminar, pero también respetando la idea del otro. Entonces, es un espacio para que también se propicien diálogo de saberes y haceres. 
La comunicación intercultural es un mecanismo de articulación social, no de dominio, porque moviliza a la comunidad sobre aspectos de interés individual, pero también de carácter mutuo y colectivo. Es vivencial y oportuna, colectiva y diversa, humana y respetuosa, incluyente y reciproca, horizontal y capaz de generar lazos de hermandad entre los diferentes actores sociales y con los elementos de la Madre Tierra.

La comunicación intercultural es aquella que se preocupa por ayudar a crear una atmósfera de cooperación y entendimiento entre las diferentes culturas, basada en la tolerancia y el deseo de aceptar lo inesperado y la flexibilidad para cambiar o adoptar alternativas, (Gaudete Comunicación, 2015).

Entonces, la comunicación intercultural es diferente a la comunicación tradicional, en el aspecto que la interculturalidad procura conocer la cultura ajena pero no desde un aspecto superficial, sino entender los fundamentos de esa cultura y aperturarse a aprender esos conocimientos y prácticas.

La Interculturalidad y la Comunicación Intercultural se ha concebido mediante la convivencia, interacción, acción de compartir valores, modo de establecer vínculos y relaciones entre las personas de diferentes culturas, como un elemento de unidad en la diversidad y el fortalecimiento de las identidades multiculturales que habitan los pueblos indígenas y afrodescendientes de Abya Yala, (Montoya, 2017, p. 1).

Además, la comunicación intercultural intenta llevarnos a un reconocimiento de la cultura propia, la que a su vez nos hace eliminar prejuicios sobre las "otras" culturas, sobre los otros conocimientos, sobre las otras prácticas, haciéndonos seres capaces de tener empatía por nuevos saberes y haceres.

"Hablar de interculturalidad, muchas veces, se hace desde el discurso, sin realmente profundizar en los grandes desafíos que presenta, tales como: compartir el poder en los diferentes espacios de toma de decisiones, propiciar la igualdad de las oportunidades, la redistribución equitativa de los recursos, la visibilización de los diferentes pueblos en las estadísticas y en la puesta en práctica de políticas y acciones con y para los pueblos que partan de sus verdaderas necesidades, analizar las relaciones entre hombres y mujeres desde la perspectiva intercultural de género y sus diferentes situaciones, experiencias, cosmovisiones y su manera de implementar la complementariedad y la paridad sin violar los derechos humanos, (Hooker, 2018).

Entonces, la comunicación intercultural debe ser ese ejercicio constante por construir, por aportar y por ser un elemento de guía y de oportunidades para los pueblos y comunidades indígenas, debe ser esa herramienta de fortalecimiento y empoderamiento que lleve al emprendimiento y entendimiento de nuevas manifestaciones culturales, en el marco del respeto, hermandad, solidaridad y en armonía con 
la Madre Tierra. La interculturalidad representa lograr la igualdad de oportunidades y permitir el mantenimiento de la diversidad y potenciar el aprendizaje con otros y de otros (Ríos, 2011).

Por lo tanto, la comunicación intercultural incluye todos lo elementos de las culturas y sus procesos de construcción, estos elementos se manifiestas de diversas maneras en cada realidad, a través de instrumentos propios que transmiten la vida de una cultura como los museos culturales, donde se encuentran inmersos los saberes y prácticas de una comunidad. La comunicación intercultural tiene el reto de transmitir todos esos elementos de manera armoniosa y respetando cada punto de vista, cada visión del mundo, cada elemento representativo que ha sido invisibilizado a lo largo de la historia de los pueblos.

\section{Hace visible lo históricamente invisible}

La transmisión de las realidades, historias, vidas, visiones y las prácticas de los pueblos y comunidades indígenas, es uno de los propósitos de la comunicación intercultural. Intenta presentar lo que no se dice, es una práctica que dice lo que se ha ocultado a lo largo de la historia.

La comunicación occidental aborda temas desde la perspectiva del comunicador y muchas veces presenta historias generalmente sesgadas y acorde a los ideales del medio y no a las realidades de los territorios y de los actores. La comunicación intercultural trata de romper con ese esquema y se muestra como un portal para visibilizar lo que ha sido mancillado históricamente.

La Comunicación, debe cuidar la información para que no lastime, que no agreda, que no denigre a los pueblos sea cual sea el contexto y la noticia, (Hooker, 2017). Además, elementalmente la comunicación intercultural muestra historias contadas por los actores, donde los principales actores son sujetos y no solamente objetos de estudio, es una apertura para los pueblos indígenas, para la UNESCO (2006), citado en (Hooker, 2018), la interculturalidad se define como la presencia e interacción equitativa de diversas culturas, y la posibilidad de generar expresiones culturales compartidas a través del diálogo y el respeto mutuo.

Entonces, la comunicación intercultural se trata de un intercambio cultural en igualdad, sin ignorar o invisibilizar las posiciones del "otro", al contrario, se busca equilibrar las diversas posiciones y, sobre todo, reconocerlas, excluyendo en todo momento el paternalismo y el victimismo, puesto que estos son elementos de exclusión y transgresión a los pueblos indígenas.

Por lo tanto, de acuerdo con Hooker (2018), la interculturalidad debe ser entendida como un vínculo entre el conocimiento y la necesidad de la comunidad y de sus 
personas en cada realidad de su vida. Es un proceso que se debe realizar desde la vida y para la vida.

\section{Discusión y conclusiones}

Ante una sociedad cambiante, el papel de la comunicación intercultural es urgente y necesaria para que se adhiera a las generaciones que actualmente viven y vienen a futuro, para asegurar una relación más humana y de derechos humanos donde se materialicen las vivencias respetando el ser diferente.

Es necesario institucionalizar e implementar el Diálogo de Saberes y Haceres desde cada uno de los espacios académicos y organizacionales para asegurar esa vivencia directa de sujeto a sujeto entre las comunidades indígenas y afrodescendientes y el mundo occidental.

Es urgente, hacer conciencia a los grandes monopolios que manejan los medios de comunicación masiva para emprender en una comunicación de cambio social, que aporte al cuido de la Madre Tierra y aporte al Buen Vivir de los Pueblos, para evitar transgredir, estigmatizar, satirizar o acusar por sus formas propias de entender y ver el mundo.

Es fundamental que los estados implementen y gestionen políticas de comunicación intercultural para asegurar una sociedad más justa, equitativa e igualitarias desde los deberes y haceres de cada ser vivo; que respete la madre tierra, que convivan e intercambie con los seres vivos de la naturaleza.

\section{Lista de referencias}

Alsina, M. (1999). La comunicación intercultural. Encontrado en http://www.portalcomunicacion.com/download/1.pdf

Atupaña, G. A. (2018). Diálogos comunitarios de saberes y haceres. Encontrado en https:// atuplan.com/2018/02/o1/dialogos-comunitarios-de-saberes-y-haceres/

Gaudete Comunicación, (3 marzo 2015). http://gaudetecomunicacion.com/?p=61

Hooker, A. (2017). El papel de la comunicación en procesos de interculturalidad. URACCAN al día, edición especial.

Hooker, A. (2018). Conferencia de Inauguración del Doctorado en Estudios InterculturaIes: "La Interculturalidad que construye Puentes", 17 de septiembre 2018, Recinto URACCAN Bilwi. 
Lasswell, H. D. (1985). Estructura y función de la comunicación en la sociedad. Sociología de la comunicación de masas. II Estructura, Funciones y Efectos. Barcelona. Gustavo Gili, 50-68.

Ríos, R. A. (2011). La comunicación intercultural. Encontrado en https://www.valencia. es/ayuntamiento/bienestarsocial.nsf/o/B251CDC7B B $45 \mathrm{C}_{9} \mathrm{~F} 8 \mathrm{FC} 125795 \mathrm{~F}_{20} 482 \mathrm{E}$ 44/\$FILE/6-AlfaM\%C3\%B3dulo2.Aurelio.pdf?OpenElement\&lang=1

Rizo, M. (2004). La comunicación como base para la interacción social. Aportaciones de la comunicología al estudio de la ciudad, la identidad y la inmigración. Contemporánea, 2(3).

Torrico, V., \& Erick, R. (2015). La comunicación" occidental". Oficios Terrestres.

Yantalema, B. (2016). El Diálogo de Saberes y Haceres desde la perspectiva de las comunidades KICHWA de la Sierra Ecuatoriana. Pluriversidad Amatatay Wasi-Ecuarunari: Quito, Ecuador. 